\title{
Existence and uniqueness of solutions for periodic-integrable boundary value problem of second order differential equation
}

Hongtu Hua ${ }^{1,2^{*}}$, Fuzhong Cong ${ }^{1}$ and Yi Cheng ${ }^{1,2}$

\section{"Correspondence:}

hongtuhua@163.com

'Fundamental Department,

Aviation University of Air Force,

Changchun, 130022, People's

Republic of China

${ }^{2}$ Institute of Mathematics, Jilin University, Changchun, 130012,

People's Republic of China

\begin{abstract}
In this paper we deal with one kind of second order periodic-integrable boundary value problem. Using the lemma on bilinear form and Schauder's fixed point theorem, we give the existence and uniqueness of solutions for the problem under Lazer type nonresonant condition.

MSC: 34B15; 34B16; 37J40

Keywords: lemma on bilinear forms; Schauder's fixed point theorem; existence and uniqueness; periodic-integrable boundary value problems
\end{abstract}

\section{Introduction and main results}

In this paper, we consider the solutions to the following periodic-integrable boundary value problem (for short, PIBVP):

$$
\begin{aligned}
& \left(p(t) x^{\prime}\right)^{\prime}+f(t, x)=0, \\
& x(0)=x(T), \quad \int_{0}^{T} x(s) \mathrm{d} s=0,
\end{aligned}
$$

where $p(t) \in C^{1}(R, R)$ is a given $T$-periodic function in $t \in R$, and $p(t)>0 ; f \in C^{1}(R \times R, R)$ is $T$-periodic in $t$.

Throughout this paper, we assume

(A1) there exist two constants $m$ and $M$ such that

$$
m \leq \frac{f_{x}(t, x)}{p(t)} \leq M
$$

for all $t \in R$ and $x \in R$;

(A2) there exists $N \in Z^{+}$such that

$$
\frac{4 \pi^{2}}{T^{2}} N^{2}<m \leq M<\frac{4 \pi^{2}}{T^{2}}(N+1)^{2} .
$$

Recently, boundary value problems with integral conditions have been studied extensively [6-10]. As we all know Lazer type conditions are essential for the existence and

\section{Springer}

(c) 2012 Hua et al: licensee Springer. This is an Open Access article distributed under the terms of the Creative Commons Attribution License (http://creativecommons.org/licenses/by/2.0), which permits unrestricted use, distribution, and reproduction in any medium, provided the original work is properly cited. 
uniqueness of periodic solutions of equations [1-4]. In [5] the existence of periodic solutions has been considered for the following second order equation:

$$
\left(p(t) x^{\prime}\right)^{\prime}+f(x, t)=0
$$

Motivated by the above works, we will consider periodic-integrable boundary value problem (1.1). The main result obtained by us is the following theorem.

Theorem 1 Assume that (A1) and (A2) are satisfied. Then PIBVP (1.1) has a unique solution.

This paper is organized as follows. Section 2 deals with a linear problem. There, using the bilinear lemma developed by Lazer, one proves the uniqueness of solutions for linear equations. In Section 3, applying the result in Section 2 and Schauder's fixed point theorem, we complete the proof of Theorem 1.

\section{Linear equation}

Consider the following linear PIBVP:

$$
\begin{aligned}
& \left(p(t) x^{\prime}\right)^{\prime}+q(t) x=0, \\
& x(0)=x(T), \quad \int_{0}^{T} x(s) \mathrm{d} s=0,
\end{aligned}
$$

here $p(t) \in C^{1}(R, R)$ is a given $T$-periodic function in $t \in R$, and $p(t)>0 ; q(t) \in C(R, R)$ is a $T$-periodic function. Assume that

(L1) there exist two constants $m$ and $M$ such that

$$
m \leq \frac{q(t)}{p(t)} \leq M
$$

for all $t \in R$. Moreover, $m$ and $M$ suit (A2).

Theorem 2 Assume that (L1) and (A2) are satisfied, then PIBVP (2.1) has only a trivial solution.

In order to prove Theorem 2, let us give some following concepts.

First, for any interval $[\alpha, \beta] \subset[0, T]$, define

$$
\begin{aligned}
\mathcal{O}_{\alpha, \beta}= & \left\{u(t) \in L^{2}(0, T): u^{\prime}(t) \text { is absolutely continuous on }[\alpha, \beta],\right. \\
& \text { and } u(t)=0 \text { for any } t \in[0, \alpha] \cup[\beta, T]\} .
\end{aligned}
$$

It is clear that $\mathcal{O}_{\alpha, \beta}$ is a linear space with the norm as follows:

$$
\|u\|=\max _{t \in[0, T]}|u(t)|+\max _{t \in[0, T]}\left|u^{\prime}(t)\right|
$$

Define a bilinear form on $\mathcal{O}_{\alpha, \beta}$ as follows:

$$
H_{\alpha, \beta}(u, v)=\int_{0}^{T}\left[p(t) u^{\prime}(t) v^{\prime}(t)-q(t) u(t) v(t)\right] \mathrm{d} t
$$


for any $u(t) \in \mathcal{O}_{\alpha, \beta}$ and $v(t) \in \mathcal{O}_{\alpha, \beta}$. Let

$$
\begin{aligned}
& \mathcal{X}_{\alpha, \beta}=\left\{x \in \mathcal{O}_{\alpha, \beta}: x=\sum_{i=N+1}^{\infty}\left(a_{i} \cos \frac{2 \pi i}{T} t+b_{i} \sin \frac{2 \pi i}{T} t\right)\right\}, \\
& \mathcal{Y}_{\alpha, \beta}=\left\{y \in \mathcal{O}_{\alpha, \beta}: y=c_{0}+\sum_{k=1}^{N}\left(c_{k} \cos \frac{2 \pi i}{T} t+d_{k} \sin \frac{2 \pi i}{T} t\right)\right\},
\end{aligned}
$$

where $N$ suits assumption (L1), and $a_{m}, b_{m}, c_{0}, c_{k}$ and $d_{k}$ are some constants. Then $\mathcal{O}_{\alpha, \beta}=$ $\mathcal{X}_{\alpha, \beta} \oplus \mathcal{Y}_{\alpha, \beta}$.

From $p(t) \in C^{1}(R, R)$ and $p(t)>0$, we can obtain that there exist two constants $M_{1}$ and $M_{2}$ such that

$$
0 \leq M_{1} \leq p(t) \leq M_{2}
$$

for all $t \in R$. Then from assumptions (L1) and (A2), we have

$$
\begin{aligned}
H_{\alpha, \beta}(x, x) & =\int_{0}^{T} p(t)\left(x^{\prime 2}(t)-\frac{q(t)}{p(t)} x^{2}(t)\right) \mathrm{d} t \\
& \geq \int_{0}^{T} p(t)\left(x^{\prime 2}(t)-M x^{2}(t)\right) \mathrm{d} t \\
& \geq \frac{2 \pi^{2} M_{1}}{T} \sum_{i=N+1}^{\infty}\left(i^{2}-(N+1)^{2}\right)\left(a_{i}^{2}+b_{i}^{2}\right) \\
& \geq 0
\end{aligned}
$$

for all $x \in \mathcal{X}_{\alpha, \beta}$, and

$$
\begin{aligned}
H_{\alpha, \beta}(y, y) & =\int_{0}^{T} p(t)\left(y^{\prime 2}(t)-\frac{q(t)}{p(t)} y^{2}(t)\right) \mathrm{d} t \\
& \leq \int_{0}^{T} p(t)\left(y^{\prime 2}(t)-m y^{2}(t)\right) \mathrm{d} t \\
& \leq \frac{2 \pi^{2} M_{2}}{T} \sum_{k=1}^{N}\left(k^{2}-N^{2}\right)\left(c_{k}^{2}+d_{k}^{2}\right)-m M_{2} T c_{0}^{2} \\
& \leq 0
\end{aligned}
$$

for all $y \in \mathcal{Y}_{\alpha, \beta}$. Thus, $H_{\alpha, \beta}$ is positive definite on $\mathcal{X}_{\alpha, \beta}$ and negative definite on $\mathcal{Y}_{\alpha, \beta}$. By the lemma in [1], we assert that if $H_{\alpha, \beta}(u, v)=0$ for all $u \in \mathcal{O}_{\alpha, \beta}$, then $v \equiv 0$.

For every $x$ on $[\alpha, \beta]$ with $x(\alpha)=x(\beta)=0$, we introduce an auxiliary function

$$
x^{\alpha, \beta}(t)= \begin{cases}x(t), & t \in[\alpha, \beta], \\ 0, & t \in[0, \alpha] \cup[\beta, T] .\end{cases}
$$

The following lemma is very useful in our proofs. 
Lemma 1 If $p(t), q(t)$ are continuous and satisfy (L1) and (A2), then the following two points boundary value problem

$$
\begin{aligned}
& \left(p(t) x^{\prime}\right)^{\prime}+q(t) x=0, \\
& x(\alpha)=x(\beta)=0
\end{aligned}
$$

has only a trivial solution.

Proof It is clear that 0 is a solution of two points boundary value problem (2.2). If $v(t)$ is a solution of problem (2.2), then $v^{\alpha, \beta}(t) \in \mathcal{O}_{\alpha, \beta}$. For any $u \in \mathcal{O}_{\alpha, \beta}$, we have

$$
\int_{\alpha}^{\beta}\left[\left(p(t) v^{\prime}(t)\right)^{\prime} u(t)+q(t) u(t) v(t)\right] \mathrm{d} t=0
$$

by using (2.2). Integrating the first terms by parts, we derive

$$
-H_{\alpha, \beta}\left(u, v^{\alpha, \beta}\right)=-\int_{0}^{T}\left[p(t) u^{\prime}(t) v^{\alpha, \beta^{\prime}}(t)-q(t) u(t) v^{\alpha, \beta}(t)\right] \mathrm{d} t=0 .
$$

By assumption (L1), $H_{\alpha, \beta}$ is positive definite on $\mathcal{X}_{\alpha, \beta}$ and negative definite on $\mathcal{Y}_{\alpha, \beta}$. These show $v^{\alpha, \beta}(t) \equiv 0$ for $t \in[0, T]$, that is, $v(t) \equiv 0$ for $t \in[\alpha, \beta]$. The proof of Lemma 1 is ended.

Proof of Theorem 2 It is clear that PIBVP (2.1) has at least one solution, for example, $x_{*} \equiv 0$. Assume that PIBVP (2.1) possesses a nontrivial solution $x^{*} \neq 0$. The proof is divided into three parts.

Case 1: $x^{*}(0)=x^{*}(T)=0$. By Lemma $1(\alpha=0$ and $\beta=T)$, PIBVP (2.1) has only a trivial solution. This contradicts $x^{*} \neq 0$.

Case 2: $x^{*}(0)=x^{*}(T)=\eta>0$. Denote

$$
S=\left\{t \in[0, T]: x^{*}(t)=0\right\}
$$

Take

$$
a=\inf _{t \in S} t \quad \text { and } \quad b=\sup _{t \in S} t
$$

From $\int_{0}^{2 \pi} x^{*}(s) \mathrm{d} s=0$, there are at least two points in the set $S$, which implies that $0<a<$ $b<2 \pi$ and $x^{*}(a)=x^{*}(b)=0$. By Lemma $1(\alpha=a$ and $\beta=b)$ the two points boundary value problem

$$
\begin{aligned}
& \left(p(t) x^{\prime}\right)^{\prime}+q(t) x=0, \\
& x(a)=x(b)=0
\end{aligned}
$$

only has a trivial solution. Hence we obtain $x^{*}(t) \equiv 0, t \in[a, b]$. By the definitions of $a$ and $b$, one has

$$
x^{*}(t)>0 \quad \text { for all } t \in[0, a) \cup(b, T] .
$$


From $\int_{0}^{T} x^{*}(s) \mathrm{d} s=0$, we get

$$
\int_{0}^{a} x^{*}(s) \mathrm{d} s+\int_{b}^{T} x^{*}(s) \mathrm{d} s=0
$$

This contradicts $\int_{0}^{a} x^{*}(s) \mathrm{d} s>0$ and $\int_{b}^{T} x^{*}(s) \mathrm{d} s>0$.

Case 3: $x^{*}(0)=x^{*}(T)=\eta<0$. This case is similar to Case 2 .

Thus, we complete the proof of Theorem 2 .

Theorem 3 If $p(t), q(t)$ are continuous and satisfy(L1) and (A2), then the following PIBVP

$$
\begin{aligned}
& \left(p(t) x^{\prime}\right)^{\prime}+q(t) x=h(t), \\
& x(0)=x(T), \quad \int_{0}^{T} x(s) \mathrm{d} s=0
\end{aligned}
$$

has a unique solution.

Proof Let $x_{* 1}(t)$ and $x_{* 2}(t)$ be two linear independent solutions of the following linear equation:

$$
\left(p(t) x^{\prime}\right)^{\prime}+q(t) x=0 .
$$

Assume that $x_{*}(t)=c_{1} x_{* 1}(t)+c_{2} x_{* 2}(t)$ is a solution of PIBVP (2.1), where $c_{1}$ and $c_{2}$ are constants. Then by the boundary value conditions of (2.1),

$$
\left\{\begin{array}{l}
\left(x_{* 1}(0)-x_{* 1}(T)\right) c_{1}+\left(x_{* 2}(0)-x_{* 2}(T)\right) c_{2}=0, \\
\int_{0}^{T} x_{* 1}(s) \mathrm{d} s c_{1}+\int_{0}^{T} x_{* 2}(s) \mathrm{d} s c_{2}=0
\end{array}\right.
$$

By Theorem 3, PIBVP (2.1) has only a trivial solution, which shows

$$
\left|\begin{array}{cc}
x_{* 1}(0)-x_{* 1}(T) & x_{* 2}(0)-x_{* 2}(T) \\
\int_{0}^{T} x_{* 1}(s) \mathrm{d} s & \int_{0}^{T} x_{* 2}(s) \mathrm{d} s
\end{array}\right| \neq 0
$$

Let $x_{\star}(t)=c_{3} x_{* 1}(t)+c_{4} x_{* 2}(t)+x_{* 0}(t)$ be a solution of PIBVP $(2.4)$, where $x_{* 0}(t)$ is a solution of the equation

$$
\left(p(t) x^{\prime}\right)^{\prime}+q(t) x=h(t) .
$$

From the boundary value conditions, we have

$$
\left\{\begin{array}{l}
\left(x_{* 1}(0)-x_{* 1}(T)\right) c_{3}+\left(x_{* 2}(0)-x_{* 2}(T)\right) c_{4}=x_{* 0}(0)-x_{* 0}(T), \\
\int_{0}^{T} x_{* 1}(s) \mathrm{d} s c_{3}+\int_{0}^{T} x_{* 2}(s) \mathrm{d} s c_{4}=\int_{0}^{T} x_{* 0}(s) \mathrm{d} s
\end{array}\right.
$$

From (2.5) constants $c_{3}, c_{4}$ are unique. Thus, PIBVP (2.4) has only one solution. 


\section{Nonlinear equations}

Let us prove Theorem 1. Rewrite (1.1) as follows:

$$
\begin{aligned}
& \left(p(t) x^{\prime}\right)^{\prime}+h(t, x) x=-f(t, 0), \\
& x(0)=x(T), \quad \int_{0}^{T} x(s) \mathrm{d} s=0,
\end{aligned}
$$

where

$$
h(t, x)=\int_{0}^{1} f_{x}(t, \theta x) \mathrm{d} \theta
$$

Define

$$
\begin{gathered}
\mathcal{O}^{*}=\left\{u(t) \in L^{2}(0, T): u^{\prime}(t) \text { is absolutely continuous on }[0, \mathrm{~T}],\right. \\
\left.u(0)=u(T) \text { and } \int_{0}^{T} u(s) \mathrm{d} s=0\right\} .
\end{gathered}
$$

Fix $y \in \mathcal{O}^{*}$, introduce an auxiliary PIBVP

$$
\begin{aligned}
& \left(p(t) x^{\prime}\right)^{\prime}+h(t, y) x=-f(t, 0), \\
& x(0)=x(T), \quad \int_{0}^{T} x(s) \mathrm{d} s=0 .
\end{aligned}
$$

To prove the main result, we need the following Lemma 2.

Lemma 2 Iff satisfies (A1) and (A2), then for any given $y \in \mathcal{O}^{*}, P I B V P(3.2)$ has only one solution, denoted as $x_{y}(t)$ and $\left\|x_{y}\right\| \leq M$.

Proof From condition (A2), it follows that

$$
m \leq \frac{h(t, y)}{p(t)} \leq M
$$

By Theorem 3, PIBVP (3.2) has only one solution $x_{y}(t)$. If $\left\|x_{y}\right\| \leq M$ does not hold, there would exist a sequence $\left\{y_{m}(t)\right\}$ such that $\left\|x_{y_{m}}\right\| \rightarrow \infty, m \rightarrow \infty$. Choose a subsequence of $\left\{h\left(t, y_{m}\right)\right\}_{m=1}^{\infty}$, without loss of generality, express as itself, such that the sequences are weakly convergent in $L^{2}(0, T)$. Denote the limit as $h_{0}(t)$. It is obvious that $h_{0}(t) \in L^{2}(0, T)$.

Because the set

$$
S=\left\{q(t) \in L^{2}[0, T]: m \leq \frac{q(t)}{p(t)} \leq M\right\}
$$

is bounded convex in $L^{2}(0, T)$, by the Mazur theorem, we have $h_{0}(t) \in S$. Hence,

$$
m \leq \frac{h_{0}(t)}{p(t)} \leq M
$$


By the Arzela-Ascoli theorem, passing to a subsequence, we may assume that

$$
x_{m}=\frac{x_{y_{m}}}{\left\|x_{y_{m}}\right\|} \rightarrow x_{0}
$$

and $x_{m}^{\prime} \rightarrow z(t)$ in $C([0, T], R)$. Thus, $x_{0}(0)=x_{0}(T)$ and $\int_{0}^{T} x_{0}(s) \mathrm{d} s=0$.

By

$$
x_{m}(t)=x_{m}(0)+\int_{0}^{t} x_{m}^{\prime}(s) \mathrm{d} s,
$$

one has

$$
x_{0}(t)=x_{0}(0)+\int_{0}^{t} z(s) \mathrm{d} s,
$$

which implies $z(t)=x_{0}^{\prime}(t)$, for any $t \in[0, T]$. Hence, $\left\|x_{0}\right\|=1$.

From PIBVP (3.2), we obtain

$$
\begin{aligned}
& \left(p(t) x_{m}^{\prime}\right)^{\prime}+h\left(t, y_{m}\right) x_{m}=\frac{-f(t, 0)}{\left\|x_{y_{m}}\right\|}, \\
& x_{m}(0)=x_{m}(T), \quad \int_{0}^{T} x_{m}(s) \mathrm{d} s=0 .
\end{aligned}
$$

This shows that $x_{0}(t)$ is a nontrivial solution of the following PIBVP:

$$
\begin{aligned}
& \left(p(t) x^{\prime}\right)^{\prime}+h_{0}(t) x=0, \\
& x(0)=x(T), \quad \int_{0}^{T} x(s) \mathrm{d} s=0 .
\end{aligned}
$$

On the other hand, by Theorem 2, PIBVP (3.4) has only zero, which leads to a contradiction. The proof of Lemma 2 is completed.

Set

$$
B_{M^{*}}=\left\{x \in \mathcal{O}^{*}:\|x\| \leq M^{*}\right\}
$$

Define an operator $F: \mathcal{O}^{*} \rightarrow \mathcal{O}^{*}$ by $F y=x_{y}$. Applying Lemma 2, $F: B_{M^{*}} \rightarrow B_{M^{*}}$.

Lemma 3 Operator $F$ is completely continuous on $\mathcal{O}^{*}$.

Proof We first prove that $F$ is continuous. Given any $\left\{y_{m}\right\} \subset \mathcal{O}^{*}$ such that $y_{m} \rightarrow y_{0} \in \mathcal{O}^{*}$. Put $u_{m}=x_{y_{m}}-x_{y_{0}}$. From the definition

$$
\begin{aligned}
& \left(p(t) u_{m}^{\prime}\right)^{\prime}+h\left(t, y_{m}\right) u_{m}=\left[h\left(t, y_{0}\right)-h\left(t, y_{m}\right)\right] x_{y_{0}}, \\
& u_{m}(0)=u_{m}(T), \quad \int_{0}^{T} u_{m}(s) \mathrm{d} s=0 .
\end{aligned}
$$


We would prove that $u_{m} \rightarrow 0$ in $C^{1}([0, T], R)$. If not, then there would be a $c>0$ such that

$$
\lim _{m \rightarrow \infty} \sup \left\|u_{m}\right\| \geq c
$$

Utilizing Lemma 2 and Arzela-Ascoli theorem, passing to a subsequence, we may assume that $u_{m} \rightarrow u_{0}$. Similar to the proof of Lemma 2, we have $u_{m}^{\prime} \rightarrow u_{0}^{\prime}$. Then

$$
\begin{aligned}
& \left(p(t) u_{0}^{\prime}\right)^{\prime}+h\left(t, y_{0}\right) u_{0}=0, \\
& u_{0}(0)=u_{0}(T), \quad \int_{0}^{T} u_{0}(s) \mathrm{d} s=0 .
\end{aligned}
$$

Moreover,

$$
m \leq \frac{h\left(t, y_{0}\right)}{p(t)} \leq M
$$

Hence, from Theorem 2, $u_{0}(t) \equiv 0$. This implies $F$ is continuous. By Lemma 2, for any bounded subset $D \subset \mathcal{O}^{*}, F(D)$ is also bounded. Hence, applying the continuity of $F$ and Arzela-Ascoli theorem, $F(D)$ is relatively compact. This shows $F$ is completely continuous on $\mathcal{O}^{*}$. The proof of Lemma 3 is completed.

Proof of Theorem 1 By Lemma 2, Lemma 3 and Schauder's fixed point theorem, $F$ has a fixed point in $\mathcal{O}^{*}$, that is, PIBVP (1.1) has a solution $x(t)$.

The following is to prove uniqueness. Let $x_{1}(t)$ and $x_{2}(t)$ be any two solutions of equation (1.1). Then $x(t)=x_{1}(t)-x_{2}(t)$ is a solution of the equation

$$
\left(p(t) x^{\prime}\right)^{\prime}+x \int_{0}^{1} f_{x}\left(t, x_{2}+\theta x\right) \mathrm{d} \theta=0
$$

Employing (A2), we have

$$
m \leq \frac{\int_{0}^{1} f_{x}\left(t, x_{2}+\theta x\right) \mathrm{d} \theta}{p(t)} \leq M .
$$

Hence by Theorem $3, x(t) \equiv 0$. The uniqueness is proved.

\section{Competing interests}

The authors declare that they have no competing interests.

\section{Authors' contributions}

Each of the authors, $\mathrm{HH}, \mathrm{FC}$ and $\mathrm{YC}$ contributed to each part of this work equally and read and approved the final version of the manuscript.

\section{Acknowledgements}

The authors are grateful to the referees for their useful comments. The research of F. Cong was partially supported by NSFC Grant (11171350) and Natural Science Foundation of Jilin Province of China (201115133). 
References

1. Lazer, AC: Application of a lemma on bilinear forms to a problem in nonlinear oscillations. Proc. Am. Math. Soc. 33, 89-94 (1972)

2. Brown, KJ, Lin, SS: Periodically perturbed conservative systems and a global inverse function theorem. Nonlinear Anal. 4, 193-201 (1979)

3. Wang, $\mathrm{H}, \mathrm{Li}, \mathrm{Y}$ : Existence and uniqueness of periodic solution for Duffing equations across many points of resonance. J. Differ. Equ. 108, 152-169 (1994)

4. Asakawa, H: Landesman-Lazer type problems for Fucik spectrum. Nonlinear Anal. 26, 407-414 (1996)

5. Cong, F: Periodic solutions for second order differential equations. Appl. Math. Lett. 18, 787-793 (2005)

6. Jankowski, T: Differential equations with integral boundary conditions. J. Comput. Appl. Math. 147, 1-8 (2002)

7. Yang, Z: Positive solutions to a system of second-order nonlocal boundary value problems. Nonlinear Anal. 62, 1251-1265 (2005)

8. Yang, Z: Existence and nonexistence results for positive solutions of an integral boundary value problem. Nonlinear Anal. 65, 1489-1511 (2006)

9. Yang, Z: Positive solutions of a second-order integral boundary value problem. J. Math. Anal. Appl. 321, 751-765 (2006)

10. Yang, Z: Existence of nontrivial solutions for a nonlinear Sturm-Liouville problem with integral boundary conditions. Nonlinear Anal. 67, 216-225 (2008)

doi:10.1186/1687-2770-2012-89

Cite this article as: Hua et al.: Existence and uniqueness of solutions for periodic-integrable boundary value problem of second order differential equation. Boundary Value Problems 2012 2012:89.

\section{Submit your manuscript to a SpringerOpen ${ }^{\circ}$ journal and benefit from:}

- Convenient online submission

- Rigorous peer review

- Immediate publication on acceptance

Open access: articles freely available online

- High visibility within the field

- Retaining the copyright to your article 